\title{
White matter deterioration in 15 months: latent growth curve models in healthy adults
}

\author{
Naftali Raz ${ }^{\mathrm{a}, *}$, Yi Qin Yang ${ }^{\mathrm{a}}$, Karen M. Rodrigue ${ }^{\mathrm{b}}$, Kristen M. Kennedy ${ }^{\mathrm{b}}$, \\ Ulman Lindenberger ${ }^{\mathrm{c}}$, Paolo Ghisletta ${ }^{\mathrm{d}}$ \\ ${ }^{a}$ Institute of Gerontology and Department of Psychology, Wayne State University, Detroit, MI, USA \\ ${ }^{\mathrm{b}}$ Center for Vital Longevity, School of Behavioral and Brain Sciences, University of Texas at Dallas, Dallas, TX, USA \\ ${ }^{\mathrm{c}}$ Max Planck Institute for Human Development, Berlin, Germany \\ ${ }^{\mathrm{d}}$ Faculty of Psychology and Educational Sciences, University of Geneva, Geneva, and Distance Learning University, Sierre, Switzerland
}

Received 29 September 2010; received in revised form 5 November 2010; accepted 18 November 2010

\begin{abstract}
The goal of the study was to examine the differences in trajectories of change in the volume of white matter hyperintensities (WMH) in healthy adults within a relatively short period. We measured volumes of periventricular and deep WMH in frontal, temporal, parietal, and occipital lobes of healthy volunteers (age 49-83) on 3 occasions, approximately 15 months apart. At baseline, 40 participants underwent magnetic resonance imaging (MRI), 37 returned for the first and 30 for the second follow-up. Latent growth curve models estimated the variance and mean change in WMH volume and examined their associations with age, sex, education, and hypertension. In both regions and for both WMH types, the positive association between volume and age was stronger among the middle-aged adults and became weaker in older ages, as a logarithmic function of age. Individual variations were present in initial WMH volume but not in WMH volume progression. Frontal deep WMH volume was greater in hypertensive participants, whereas lower education was associated with greater posterior deep WMH volume. Thus, white matter of healthy middle-aged and older adults undergoes significant regional deterioration in a relatively short period, and is negatively affected by vascular risk and lower educational level.
\end{abstract}

(C) 2012 Elsevier Inc. All rights reserved.

Keywords: Aging; Brain; MRI; Longitudinal; White matter hyperintensities; Structural equations models

\section{Introduction}

Human brain undergoes significant structural transformations throughout the life span (Raz and Kennedy, 2009). Among the most common changes are white matter alterations that appear as areas of increased signal intensity on T2-weighted magnetic resonance (MR) images, white matter hyperintensities (WMH). WMH reflect diverse neurobiological and neuropathological phenomena, such as ischemic lesions, microbleeds, demyelination, and ex-

\footnotetext{
* Corresponding author at: Wayne State University, Institute of Gerontology and Department of Psychology, 87 East Ferry St., 226 Knapp Building, Detroit MI 48202, USA. Tel.: +1 313577 2297; fax: +1 508 2565689.

E-mail address: nraz@wayne.edu (N. Raz).
}

pansion of perivascular spaces (Pantoni and Garcia, 1997), and are associated with deficits in selected cognitive functions (Gunning-Dixon and Raz, 2000).

The evidence about age-related increase in $\mathrm{WMH}$ and their effect on cognition comes mostly from cross-sectional studies, and understanding of the WMH time course requires a longitudinal approach (Appelman et al., 2009). Longitudinal studies conducted to date show that on average, in middleaged and older adults, WMH expand or multiply within 2 (Veldink et al., 1998), 3 (Schmidt et al., 1999), 4 (Maillard et al., 2009), 5 (Martin et al., 1997), 6 (Schmidt et al., 2003), or 8 (Kraut et al., 2008) years after initial assessment. Those studies however, focused on the mean change, and did not address an important question of individual differences in WMH proliferation. Moreover, most prior studies used rat- 
ing scales, which are coarse and modestly reliable, prone to ceiling effects in healthy populations, and less sensitive to the effects of vascular risk than volumetric methods (Gouw et al., 2008).

Thus, the main goal of this study was to examine shortterm change in WMH volume and individual differences in WMH progression according to location (anterior vs. posterior) and type (deep vs. periventricular) in healthy adults screened for cardiovascular disease, diabetes, and depression. Conducting 3 waves of measurements allowed us to examine the shape of longitudinal trends and to test for acceleration and deceleration effects. An important aspect of our approach was the application of statistical methodology that enables quantification of individual differences in addition to assessment of possible nonlinear mean change (latent growth curve modeling [LGCM]; McArdle and Nesselroade, 2003). In the past, we have applied that methodology successfully to analyses of neuroanatomical changes across time (Raz et al., 2005, 2008, 2010). LGCM has several advantages over traditional analyses as it allows estimation of mean change and variability in change within the same model, and accounts for unreliability of the measured variables, by estimating separately a residual source of variance vis-à-vis variances in the intercept and in change.

\section{Methods}

\subsection{Participants}

Forty healthy volunteers (age $63.30 \pm 9.26,49-83$ years old; 24 women) from the metropolitan Detroit area enrolled in the study. The detailed description of the sample is available in the previous publication (Raz et al., 2010). The majority of participants (80\%) were Caucasians, and the rest were African-Americans. The majority (75\%) had a college degree, a Mini Mental State Examination (MMSE; Folstein et al., 1975) score of at least 26 (and for 95\% — above 27), and a depression questionnaire (CES-D; Radloff, 1977) score below 13. Trained technicians measured systolic and diastolic blood pressure with an analog mercury sphygmomanometer with a standard brachial cuff on 3 different days, once from each arm. Thus, the blood pressure readings in this study constituted an average of 6 measures. Hypertensive participants were taking standard antihypertensive medications. Of 40 participants who attended the baseline assessment, 37 returned for the first follow-up visit after a delay of about 16 months, and 30 underwent the third measurement (second 15-month follow-up). The average interval between baseline and the first follow-up was 15.93 ( \pm 1.85 ) months, while between the 2 follow-ups it was 15.45 ( \pm 2.76 ) months. See Supplementary Table S1 for a more detailed sample description.

\subsection{MRI acquisition}

Magnetic resonance (MR) images were acquired on the same 1.5 Tesla scanner (Siemens Medical Systems, Erlangen, Germany) on all 3 occasions, using a fluid attenuated inversion recovery (FLAIR) turbo spin echo (TSE) sequence with 56 contiguous coronal slices, matrix $=256 \times 256$, field of view $($ FOV $)=192 \mathrm{~mm} \times 192 \mathrm{~mm}$, voxel size was $0.8 \times 0.8 \times 3.0$ $\mathrm{mm}^{3}$, repetition time $(\mathrm{TR})=8000 \mathrm{~ms}$, echo time $(\mathrm{TE})=111$ $\mathrm{ms}$, inversion time $(\mathrm{TI})=22.60 \mathrm{~ms}$, flip angle $(\mathrm{FA})=180^{\circ}$.

\subsection{Demarcation and measurement of WMH}

White matter hyperintensities were measured on the frontal, parietal, temporal, and occipital lobes, with deep subcortical white matter (DW) and periventricular (PV) objects measured separately. The volume of WMH of each type in each region was calculated as a sum of the areas of hyperintensities multiplied by the slice thickness. Interrater reliability (intraclass correlation; ICC) exceeded 0.90 for all regions and all types of WMH. Demarcation and tracing rules are available in Supplementary Material (Fig. S1).

\subsection{Statistical analyses}

To analyze change in WMH volumes we specified the LGCM for the relations between WMH volumes and chronological age, tested a separate LGCM for each type of WMH in both areas, and then analyzed all 4 volumes together, in a multivariate extension of the LGCM, which allows testing the relations between the $4 \mathrm{WMH}$ volumes considered here.

\section{Results}

WMH were ubiquitous in the frontal lobes, but not in the other brain regions. Because the majority of participants (28 out of 40) evidenced no temporal lobe $\mathrm{WMH}$, that region was not included in the analyses. In addition, $10 \%$ of the participants had no parietal WMH on at least 1 occasion, although only 1 had no occipital WMH. To minimize the number of zero-WMH cases, we combined the parietal and occipital regions in a posterior region of interest (ROI) and compared it with the frontal ROI.

All measures showed high stability of individual differences across occasions. Between the baseline and the first follow-up, coefficients of stability were $r=0.95,0.88,0.91$, and 0.82 for frontal $\mathrm{PV}$, frontal $\mathrm{DW}$, posterior $\mathrm{PV}$, and posterior DW WMH volumes, respectively. Between the first and second follow-ups, the coefficients of stability were even higher: $r=0.98$ for frontal PV, 0.91 for frontal DW, 0.97 for posterior PV WMH, and 0.94 for posterior DW. The LGCM results (summarized in Table 1) revealed significant increase in the volume of frontal and posterior periventricular as well as deep WMH.

Individual differences in baseline volume estimated at 49 years of age (the lower age limit of the sample) were significant for deep and periventricular WMH in both ex- 
Table 1

Analyses of longitudinal change in the WMH volume: latent growth curve models

\begin{tabular}{|c|c|c|c|c|}
\hline \multirow[t]{2}{*}{ ROI } & \multicolumn{2}{|l|}{ Mean } & \multicolumn{2}{|l|}{ Variance } \\
\hline & Intercept & Change & Intercept & Residual \\
\hline \multicolumn{5}{|l|}{ Frontal } \\
\hline PV & $6.158(0.237)$ & $0.362(0.083)$ & $0.441(0.101)$ & $0.037(0.006)$ \\
\hline DW & $3.749(0.468)$ & $0.582(0.161)$ & $1.620(0.383)$ & $0.096(0.018)$ \\
\hline \multicolumn{5}{|c|}{ Posterior (parietal and occipital) } \\
\hline PV & $12.434(0.683)$ & $0.845(0.242)$ & $3.119(0.730)$ & $0.358(0.062)$ \\
\hline DW & $10.179(0.915)$ & $0.819(0.331)$ & $3.609(0.932)$ & $1.225(0.216)$ \\
\hline
\end{tabular}

All effects are significant $(p<0.05)$. Variances in change were not significant.

Key: DW, deep subcortical white matter; PV, periventricular; ROI, region of interest; WMH, white matter hyperintensities.

amined regions, but no variance in volume change was detected in any region. Across WMH types and regions, the expansion was logarithmically related to age. The steepest increase in strength of that association occurred in the fifth decade (see Fig. 1). Note that 4 participants had no deep frontal and 1 had no deep posterior WMH at any occasion, 2 additional participants had no posterior or frontal deep $\mathrm{WMH}$ at baseline, and 1 participant had a zero deep WMH volume at the first follow-up. Those data points (15 out of 107) were excluded from model fitting because they were
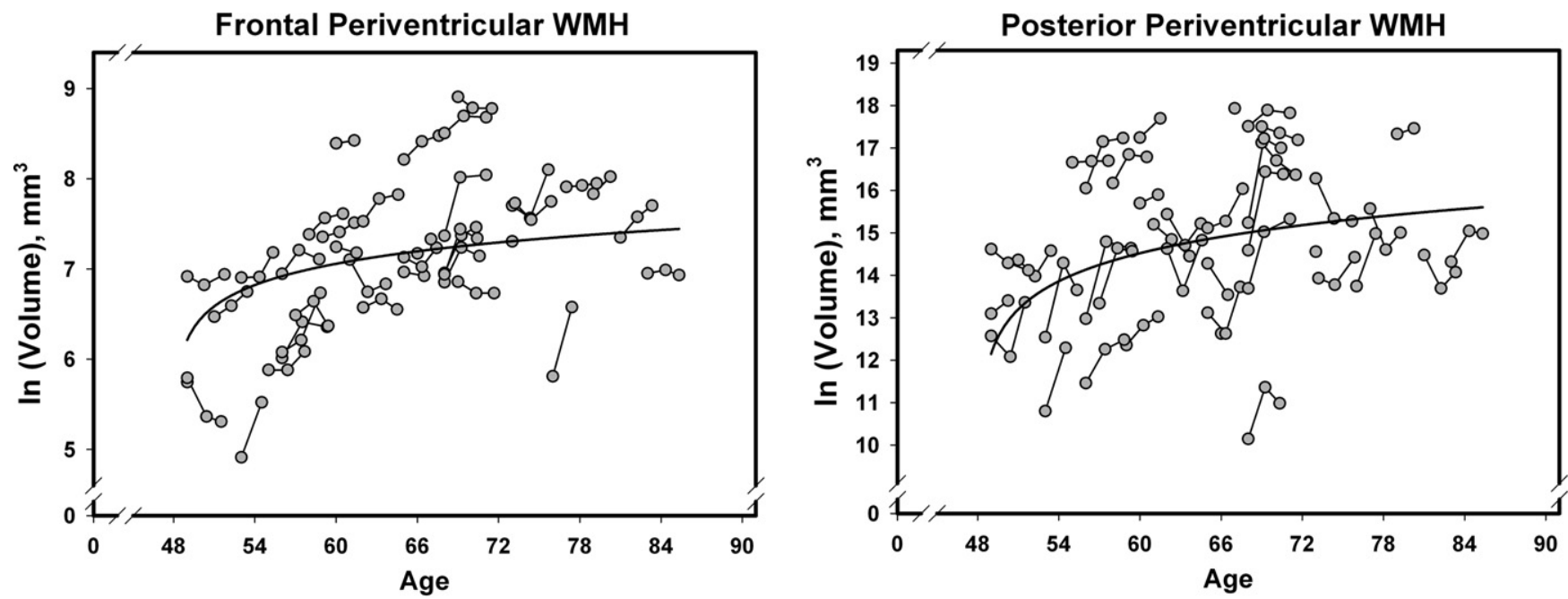

Frontal Deep WMH
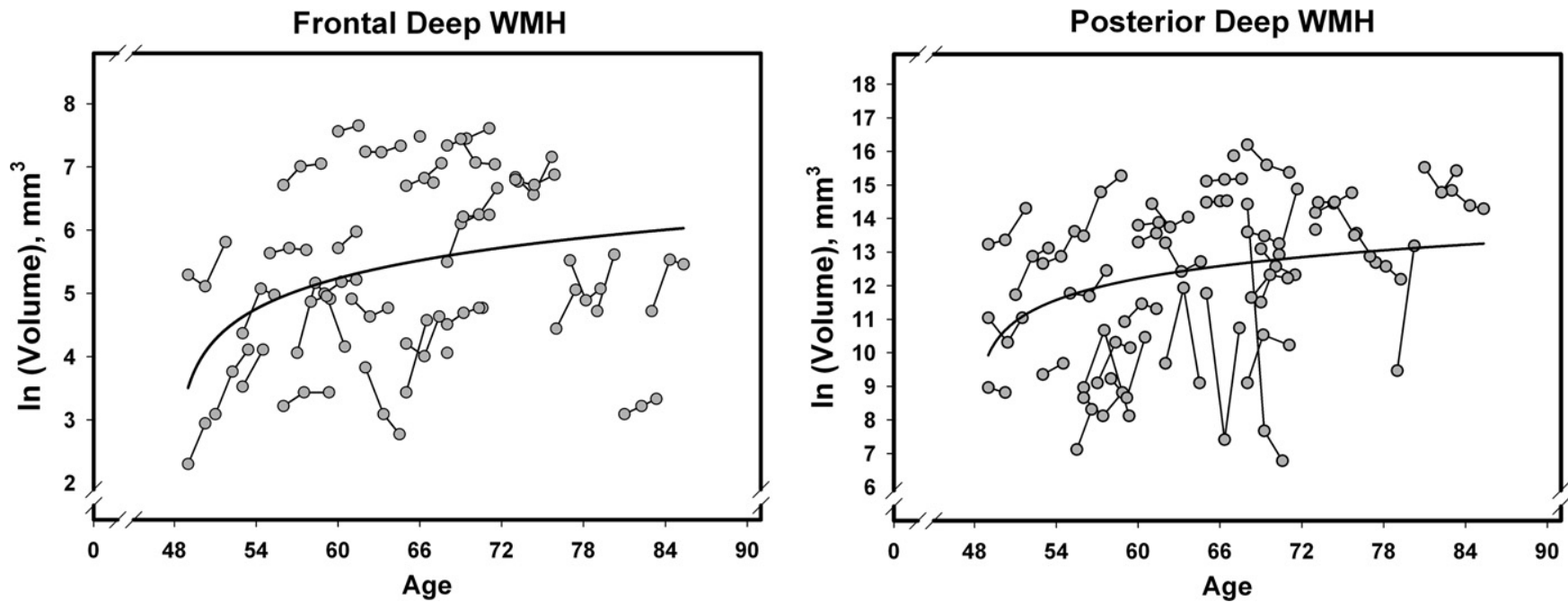

Fig. 1. Plots of individual trajectories in the volume of periventricular and deep white matter hyperintensities (WMH) in frontal and posterior regions. Natural log-transformed volumes are in $\mathrm{mm}^{3}$. For discussion of zero values (outliers removed), see text. Curve-fitting details are in Supplementary Material. 
clear outliers ( $>2.5 \mathrm{SD}$ below the mean). In the multivariate extension of the LGCM, we analyzed all $4 \mathrm{WMH}$ volumes together, to ascertain the degree of relations among them. Correlation among all the volumes at baseline did not differ from each other and could be set equal without loss of fit to $r=0.44$, i.e., almost $20 \%$ of the shared variance in initial volume across all types of $\mathrm{WMH}$ and brain regions.

To examine the potential modifiers of initial WMH volumes and to account for individual differences we repeated the LGCM analyses with the following covariates - sex, education, and hypertension. The analyses revealed only 2 significant effects. Having high blood pressure or a history of hypertension was associated with greater deep WMH volume in the frontal lobe: $\beta=1.09, p<0.05,13 \%$ of variance explained. Having lower education was associated with a greater baseline volume in posterior deep WMH: $\beta=$ $-0.29, p<.05,12 \%$ of variance explained. Analysis of sex differences revealed no effect. We also examined 2 variables that could confound longitudinal results: duration of testing interval between the first and the second follow-ups and selective participant attrition between the follow-up assessments. Variable delay had no effect on the estimated parameters. The volume of periventricular WMH was unrelated to the dropout status ( $p>0.6$ for all comparisons), although 3 participants who dropped out after the baseline had significantly larger deep WMH than those who continued: Mann-Whitney test $\chi^{2}(1)=4.39, p=.036$ for the frontal and $\chi^{2}(1)=3.90, p=.048$ for the posterior deep WMH. However, there was no difference in any WMH volumes between participants who completed 1 or 2 follow-up assessments: Mann-Whitney $p$ 's ranging from 0.29 to 0.89 , all not significant, i.e., in the longitudinal part of the study, there was no selection bias based on WMH burden.

\section{Discussion}

The presented results demonstrate that not only WMH are common in adults selected for better than average health, but the WMH volume expands within a relatively short period of slightly more than a year. We replicated the predilection of WMH to the frontal lobes in normal aging (e.g., Raz et al., 2003), and age-related increase in WMH volume therein (Raz et al., 2007). To the best of our knowledge, this is the shortest term deterioration in white matter structure reported in the extant literature. We observed no significant heterogeneity in WMH expansion. However, with few occasions, power to detect individual differences in change is low (Hertzog et al., 2008). Vascular risk and education explained only some of the baseline variance in deep WMH, but did not affect the course of expansion.

Several limitations qualified the interpretation of the findings. Selection of participants for optimal health precludes generalization to a broad population and compresses heterogeneity. The burden of $\mathrm{WMH}$ was light, and there were relatively few participants older than 70, who could have had larger WMH and steeper trajectories of expansion. Although we attempted to differentiate among types and locations of WMH, our classification was still rather coarse, as in healthy adults, attempts at fine differentiation of WMH produce untenably small numbers that would have precluded meaningful statistical analyses. In spite of excellent reliability and high stability of individual differences across measurement occasions, the appearance of small irregular objects such as WMH is affected by between-occasion variation in participant's positioning, which in a 2D fluid attenuated inversion recovery (FLAIR) scan with relatively few thick slices cannot be corrected through digital reformatting. In conclusion, white matter of healthy middle aged and older adults shows regional deterioration over relatively short time. Vascular risk is associated with greater baseline WMH volume, but not with the expansion rate.

\section{Acknowledgements}

This project was supported by Grants R37 AG-011230 and R03 AG-024630 from the National Institute on Aging, USA.

\section{Disclosure statement}

The authors have no actual or potential conflicts of interest including any financial, personal or other relationships with other people or organizations within 3 years of beginning this work. Appropriate approval and procedures were used concerning human subjects.

\section{Appendix A. Supplementary data}

Supplementary material associated with this article can be found, in the online version, at doi:10.1016/j.neurobiolaging. 2010.11.018.

\section{References}

Appelman, A.P.A., Exalto, L.G., van der Graaf, Y., Biessels, G.J., Mali, W.P.T.M., Geerlings, M.I., 2009. White matter lesions and brain atrophy: More than shared risk factors? A Systematic Review. Cerebrovasc. Dis. $28,227-242$.

Gunning-Dixon, F.M., Raz, N., 2000. The cognitive correlates of white matter abnormalities in normal aging: A quantitative review. Neuropsychology 14, 224-232.

Folstein, M.F., Folstein, S.E., McHugh, P.R., 1975. "Mini-mental state". A practical method for grading the cognitive state of patients for the clinician. J. Psychiatr. Res. 12, 189-198.

Gouw, A.A., Van Der Flier, W.M., Van Straaten, E.C.W., Pantoni, L., Bastos-Leite, A.J., Inzitari, D., Erkinjuntti, T., Wahlund, L.O., Ryberg, C., Schmidt, R., Fazekas, F., Scheltens, P., Barkhof, F., 2008. Reliability and sensitivity of visual scales versus volumetry for evaluating white matter hyperintensity progression. Cerebrovasc. Dis. 25, 247253.

Hertzog, C., Oertzen, T., Ghisletta, P., Lindenberger, U., 2008. Evaluating the power of latent growth curve models to detect individual differences in change. Struct. Equ. Modeling. 15, 541-563. 
Kraut, M.A., Beason-Held, L.L., Elkins, W.D., Resnick, S.M., 2008. The impact of magnetic resonance imaging-detected white matter hyperintensities on longitudinal changes in regional cerebral blood flow. J. Cereb. Blood Flow Metab. 28, 190-197.

Maillard, P., Crivello, F., Dufouil, C., Tzourio-Mazoyer, N., Tzourio, C., Mazoyer, B., 2009. Longitudinal follow-up of individual white matter hyperintensities in a large cohort of elderly. Neuroradiology 51, 209220.

Martin, C., van Swieten, J., Sever, A., Scheltens, P., Pieterman, H., Breteler, M., 1997. Change in white matter lesions in 60 healthy elderly over a 5-year period. J. Neurol. 244, S23.

McArdle, J.J., Nesselroade, J.R., 2003. Growth curve analysis in contemporary psychological research, in: Schinka, J., Velicer, W. (Eds.), Comprehensive Handbook of Psychology, Volume 2 Research Methods in Psychology. Wiley, New York, NY, pp. 447-480.

Pantoni, L., Garcia, J.H., 1997. Pathogenesis of leukoaraiosis: A review. Stroke 28, 652-659.

Radloff, L.S., 1977. The CES-D scale: A self-report depression scale for research in the general population. Appl. Psychol. Meas. 1, 385-401.

Raz, N., Kennedy, K.M., 2009. A systems approach to age-related change: Neuroanatomic changes, their modifiers, and cognitive correlates, in: Jagust, W., D’Esposito, M., (Eds.), Imaging the Aging Brain. Oxford University Press, New York, NY, pp. 43-70.

Raz, N., Lindenberger, U., Ghisletta, P., Rodrigue, K.M., Kennedy, K.M., Acker, J.D., 2008. Neuroanatomical correlates of fluid intelligence in healthy adults and persons with vascular risk factors. Cereb. Cortex 18, $718-726$.

Raz, N., Lindenberger, U., Rodrigue, K.M., Kennedy, K.M., Head, D., Williamson, A., Dahle, C., Gerstorf, D., Acker, J.D., 2005. Regional brain changes in aging healthy adults: General trends, individual differences and modifiers. Cereb. Cortex 15, 1676-1689.

Raz, N., Rodrigue, K.M., Acker, J.D., 2003. Hypertension and the Brain: Vulnerability of the Prefrontal Regions and Executive Functions. Behav. Neurosci. 117, 1169-1180.

Raz, N., Rodrigue, K.M., Kennedy, K.M., Acker, J.D., 2007. Vascular health and longitudinal changes in brain and cognition in middle-aged and older adults. Neuropsychology 21, 149-157.

Raz, N., Ghisletta, P., Rodrigue, K.M., Kennedy, K.M., Lindenberger, U., 2010. Trajectories of brain aging in middle-aged and older adults: Regional and individual differences. NeuroImage 51, 501-511, March 8, 2010. [Epub ahead of print].

Schmidt, R., Enzinger, C., Ropele, S., Schmidt, H., Fazekas, F., 2003. Progression of cerebral white matter lesions: 6-year results of the Austrian Stroke Prevention Study. Lancet 361, 2046-2048.

Schmidt, R., Fazekas, F., Kapeller, P., Schmidt, H., Hartung, H.P., 1999. MRI white matter hyperintensities: Three year follow-up of the Austrian Stroke Prevention Study. Neurology 53, 132-139.

Veldink, J.H., Scheltens, P., Jonker, C., Launer, L.J., 1998. Progression of cerebral white matter hyperintensities on MRI is related to diastolic blood pressure. Neurology 51, 319-320. 